\title{
Benign ovarian cysts in reproductive-age women undergoing assisted reproductive technology treatment
}

\author{
Guy Rofe, Ron Auslender, Martha Dirnfeld
}

Division of Fertility - In Vitro Fertilization, Department of Obstetrics and Gynecology, Carmel Medical Center, Faculty of Medicine, Technion Haifa, Israel

Email: guyrofe@gmail.com

Received 28 July 2013; revised 21 August 2013; accepted 26 August 2013

Copyright (C) 2013 Guy Rofe et al. This is an open access article distributed under the Creative Commons Attribution License, which permits unrestricted use, distribution, and reproduction in any medium, provided the original work is properly cited.

\begin{abstract}
The scope of this review is to focus on the management of benign ovarian cyst in the reproductive-age group of women undergoing ART. Ovarian cysts are a common occurrence in this patient population. The differential diagnosis includes functional cysts, dermoid cysts, endometrioma. The appropriate evaluation includes medical history and physical examination, laboratory tests and imaging. The treatment options include conservative follow-up, medical treatment and surgery. This review will explore the differential diagnosis, appropriate work-up and treatment options to the various cyst types encountered.
\end{abstract}

Keywords: Ovarian Cyst; Assisted Reproductive Technology; Reproductive Age

\section{INTRODUCTION}

The scope of this review is to focus on the management of benign ovarian cyst in the reproductive-age group of women undergoing ART.

In relative frequency, functional cysts account for about $24 \%$ of all ovarian cysts, benign cysts $70 \%$ and malignant $6 \%$ [1]. The great majority of ovarian cysts occur in reproductive-age patients and most of them are benign [2]. Up to $4 \%$ of reproductive-age women will have an ovarian cyst in the luteal phase [3]. Cyst formation may appear during GnRH agonist treatment [4]. Therefore, it is common to encounter patient undergoing assisted reproductive technology (ART) with a concomitant ovarian cyst. The question arises as to the adequate diagnosis and management.

Although an ovarian mass may represent a variety of Ultrasonographic and pathological findings, there are three main and common types of benign ovarian cysts encountered in the reproductive-age patients: functional cysts, benign cystic teratoma (dermoid) cysts and endometioma $[5,6]$. Each type has unique characteristics that usually enable achieving the correct diagnosis and management.

An ovarian cyst diagnosed during the reproductive age is rarely malignant. Nevertheless, as a rule, a finding of an ovarian cyst $>5 \mathrm{~cm}$, should be followed up and if persistent, a workup should include a medical history and physical examination. Imaging tests include ovarian echography. A computed tomography scan (CT scan) is usually performed if the cyst is not clear and/or shows invaginations and solid areas. Laboratory test and markers should be performed if findings are suspicious to rule out malignancy.

We note that a possible relationship between prolonged use of clomiphene citrate and borderline or invasive ovarian cancer was reported in patients with an ovarian mass and a history of prolonged exposure (above 12 cycles) [7]. Further studies are requested to confirm these findings.

When evaluating a patient with an ovarian cyst undergoing ART treatment, in addition to the routine work-up of any woman with a similar finding, the physician should assess her ovarian reserve. After completing the appropriate work-up and reaching a diagnosis and after assessing the ovarian reserve, the physician should be able to tailor the best treatment option for the specific patient.

\section{DIFFERENTIAL DIAGNOSIS}

Differential diagnosis of any adnexal mass should include gynecologic and non-gynecologic sources. When evaluating an ovarian cyst the differential diagnosis includes both benign and malignant lesions [8]. At this age group, the vast majority of ovarian masses are benign however if a suspicion of malignancy arises, management should continue appropriately. 
Benign ovarian cysts include:

- functional cysts,

- follicular cysts;

- cysts following GnRH agonist treatment;

- corpus luteum cysts;

$\circ$ theca lutein cysts.

- benign cystic teratoma (Dermoid),

- endometrioma,

- serous cystadenoma,

- mucinous cystadenoma.

Functional cysts are benign and usually asymptomatic and therefore do not require any treatment. Follow-up and repeated scans will usually confirm spontaneous resolution of the cysts. This is not the case when the cyst is large and may interfere with fertility treatments and in vitro fertilization (IVF).

The formation of cysts during IVF treatment may be of no clinical significance or may negatively influence its outcome [4]. Transvaginal Ultrasonographic-guided cyst aspiration is often performed in cases with cysts appearing during GnRH agonist treatment before starting ovarian stimulation.

Benign cystic teratoma (dermoid) cyst is the most common ovarian tumor in women in the second and third decade of life [9] and as a rule is benign. Since torsion is not uncommon, surgical management was often advised with a view of making a definitive diagnosis during the de-torsion procedure and remove the cyst while preserving ovarian healthy tissue.

Recently, a more conservative approach has been suggested regarding dermoid cysts. Evidence suggests that a dermoid cyst, especially smaller than $6 \mathrm{~cm}$, can be followed conservatively with little risk of complications [10-12]. If cysts are not growing, removal of a dermoid cyst can be deferred after family planning is resumed.

Endometrioma is usually a sign for more advance endometriosis which is a cause of infertility. According to the new (European Society of Human Reproduction and Embryology) ESHRE guidelines [13,14], surgery should not be performed before ART only in the aim of improving pregnancy rate. Surgery should be considered before ART for tissue diagnosis, reducing the risk of infection after oocyte retrieval and improve accessibility of follicles.

It is important to notice these two recent reviews that explored the relationship between endometriosis and infertility. In the first, the authors found no adverse effect of endometrioma on IVF cycle outcome $[15,16]$. In the second, the authors concluded that the presence of severe endometriosis (stage III/IV) is associated with poor implantation and clinical pregnancy rates in women undergoing IVF treatment. This coincides with the new ESHRE guidelines regarding the management of endometrioma before ART treatments as will be discussed later.

\section{DIAGNOSIS}

\subsection{Medical History \& Physical Examination}

Any evaluation of a patient with an ovarian cyst should include a thorough medical history and a complete physical examination in the search of warning signs of malignancy. The medical history should focus of risk factors and red flags for malignancy. Symptoms such as abdominal bloating, abdominal and pelvic pain, and early satiety and appetite changes should alert the physician to the presence of malignancy and the appropriate steps should be taken $[17,18]$. It is also important to search for symptoms suggesting endometriosis, especially in this cohort of patients - reproductive-age women with infertility [19].

The physical examination should include an abdominal and pelvic examination including a bimanual pelvic examination. One should look for alerting signs such as an irregular, solid, fixed and nodular mass or a bilateral finding. The presence of ascites should warn us about the possibility of malignancy.

The physician should remember that physical examination has poor sensitivity for detecting ovarian masses $(15 \%-51 \%)$ [18,20]. In addition, one should remember that in this age group it is not uncommon to find the above warning sign with benign conditions such as endometriosis which can present with bilateral findings and irregularity on pelvic examination and ascites.

\subsection{Laboratory Tests}

In the settings of a benign ovarian mass in a reproductive-age group, there is no added value in measuring CA-125 [21-23]. CA-125 in the reproductive-age group rises in numerous conditions such as fibroids, endometriosis, adenomyosis and pelvic infections and during normal menstrual cycle. This makes CA-125 an unreliable marker for differentiating benign from malignant masses and not helpful at all in the differential diagnosis of benign cysts [21-23].

Serum levels of CA-125 are not necessary when the Sonographic appearance of the cyst is simple-thin walled, clear fluid, no intracystic structures and lees then $50 \mathrm{~mm}$ in diameter [23-26].

Anti-mullerian hormone $(\mathrm{AMH})$ is a relatively new marker of ovarian reserve and considered the most accurate one available today. A serum AMH level above 0.5 $\mathrm{ng} / \mathrm{mL}$ is consistent with good ovarian reserve, while lower levels suggest the presence of a depleted ovarian follicle pool. Levels of serum AMH may provide guidance as to the appropriate management of the patient with an ovarian cyst [27-31]. 
Reduced ovarian reserve as measure by serum levels of AMH may contraindicate surgical management in the specific patient.

Pre-operative and post-operative AMH levels may be used as a tool for evaluating ovarian damage after surgery [27-31].

\subsection{Ultrasonography}

The most widely used imaging modality is gray-scale, high frequency transvaginal ultrasonography. In asymptomatic and reproductive age group, this is the imaging modality of choice [32].

Ultrasonographic examination will provide us with the size, consistency, laterality and intracystic structures (septations, nodules, papillary extensions) of the cyst. These findings, in correlations with the physical examination will provide us with a deeper insight as to correct diagnosis.

Benign cystic teratoma (Dermoid) in characterized by fat-fluid level and intracystic floating balls, shadowing echogenicity, diffuse or regional high amplitude echoes, dermoid mesh and tip of the iceberg sign [33]. When two or more characteristic sign are present, the diagnosis of dermoid cyst can be made with a high positive predictive value [33].

Endometrioma is sonographicaly characterized by ground glass echogenicity and one to four compartments and no papillary structures with detectable blood flow [34].

Color Doppler alone does not improve diagnostic evaluation [35-37], but the combination of color flow mapping with $3 \mathrm{D}$ imaging may improve sensitivity in complex masses [38,39].

\subsection{Other Imaging Modalities}

The role of computed tomography (CT) scan and magnetic resonance imaging (MRI) in the evaluation of a suspected mass in reproductive-age women is reserved for cases in which suspicion for malignancy arises.

\section{TREATMENT OPTIONS}

The treatment option includes conservative follow up, medical treatment and surgical management.

\subsection{Conservative Management}

Conservative management can be undertaken with women with small and simple ovarian cysts (less than 50 $\mathrm{mm}$ in diameter, thin walled, clear fluid, no itracystic structures). This group of women need no follow up, since the cysts are almost invariably physiological and will regress spontaneously within 3 menstrual cycles [40].
A consensus statement published by the Society of Radiologists in Ultrasound concluded that asymptomatic simple cysts with a diameter of $30-50 \mathrm{~mm}$ in diameter do not require any follow-up, cysts with a diameter of 50 - $70 \mathrm{~mm}$ require follow-up, and cysts more than $70 \mathrm{~mm}$ in diameter should be considered for either further imaging or surgical intervention due to difficulties in examining the entire cyst [41].

The course of pregnancy of patients with dermoid and other benign ovarian cysts, including perinatal outcomes, is favorable. The cysts should be managed conservatively if possible with routine ultrasound follow-up during the pregnancy since complications are extremely rare [42].

\subsection{Medical Management}

The use of combined oral contraceptives has been proposed for the treatment of functional ovarian cysts. According to the Cochrane review of the effects of the oral contraceptive pill in the treatment of functional ovarian cysts, there was no earlier resolution in the treatment group compared to the control group [43]. However, these trials were small with significant heterogeneity. It has been shown that the use of combined oral contraceptives may reduce the risk of cyst occurrence [43-45]. Therefore, combined oral contraceptives are still a possible treatment option for already existing functional cyst.

There is no medical treatment for benign cystic teratoma (dermoid) except for expectant follow up.

Medical treatment for endometriosis includes oral contraceptives, progesterone, GnRH agonists or antagonists. The problem with this treatment options is that they impair fertility and though are counter-effective in patients seeking fertility treatments [46]. Some of the treatments are useful prior to starting stimulation for IVF, increase in the implantation rate in stages III and IV endometriosis [15, 46-49].

\subsection{Surgical Management}

Surgical management should be offered when tissue diagnosis is necessary or when the ovarian cyst interferes with oocyte retrieval or ovarian stimulation. When considering surgery in reproductive-age patient, the ovarian reserve should be assessed prior to the procedure and if severely reduced surgery may be deferred in order to further explore other treatment options.

Before performing surgery, we should discuss with the patient the issue of ovarian reserve compromise after surgery and its implication on future fertility.

Ovarian cysts that persist or increase in size after several menstrual cycles are unlikely to be functional [5052], for example mature cystic teratoma (dermoid cysts) has been shown to grow over time, increasing the risk of pain and ovarian accidents (torsion, rupture) [10,53]. 
Surgical management is therefore usually appropriate in these settings.

When encountering an endometrioma in women undergoing ART, the new ESHRE guidelines conclude that surgery should not be performed before ART only in the aim of improving pregnancy rate but for symptoms improvement. Surgery should be considered before ART for tissue diagnosis, reducing the risk of infection after oocyte retrieval and improve accessibility of follicles. The guidelines also conclude that if surgery is performed, cystectomy is the best option of treatment rather than aspiration or ablation $[13,14]$.

When surgical management is indicated, the laparoscopic approach should be undertaken. Laparoscopic surgery for ovarian masses presumed to be benign is associated with lower postoperative morbidity and shorter recovery time and is preferred to laparotomy in suitable patients [54-57]. In a systematic review, that included six randomized controlled trials comparing the laparoscopic approach to laparotomy for ovarian cysts has shown reduced febrile morbidity, less postoperative pain, lower rates of postoperative complications, earlier discharge from hospital and lower overall cost [58]. It is also costeffective because reduced hospitalization time and earlier return to work [59].

When performing laparoscopic surgery for the management of a benign ovarian cyst, complete removal of the capsule should be performed. Aspiration alone, either laparoscopicaly or vaginaly, is less effective and carries a high rate of recurrences $(46 \%-84 \%)[60,61]$.

\section{CONCLUSIONS}

Ovarian cyst is a common occurrence in the reproductive-age group. Functional ovarian cysts are physiologic and usually resolve spontaneously within a couple of menstrual cycles. Combined oral contraceptives may be used to prevent the occurrence of these cysts; however, they do not accelerate cyst resolution.

Ovarian neoplasms are usually benign in women of reproductive age. The risk of an ovarian mass being in this age group is exceedingly small.

Measurement of CA-125 is not helpful in distinguishing between benign and malignant ovarian masses, reproductive-age women.

For functional cyst, conservative management is adequate since most cases will resolve spontaneously. There is no proven advantage in the use of combined oral contraceptives. Surgery will be reserved for cases of big or symptomatic functional ovarian cyst.

For benign cystic teratoma, surgical management is indicated when the cyst is growing or to provide better accessibility to follicle during oocyte retrieval.

For endometrioma, it is recommended not to perform surgery unless in need of tissue diagnosis or performing surgery for another reason or to improve accessibility to follicle during oocyte retrieval.

Laparoscopy is commonly used to remove benign cysts above $60-70 \mathrm{~mm}$ in diameter.

Simple Ultrasonography, and if indicated, more advanced imaging techniques such as MRI enable expectant management and follow-up of ovarian cysts in women of reproductive age before deciding on surgical management. Successful introduction of Assisted Reproductive technologies and novel fertility preservation strategies have led to more awareness about the importance of ovarian reserve and its preservation.

\section{REFERENCES}

[1] Helm, C.W. (2012) Ovarian Cysts, Medscape. http://emedicine.medscape.com/article/255865-over view\#showall

[2] ACOG Practice Bulletin (2007) Management of adnexal masses. Obstetrics and Gynecology, 110, 201-214.

[3] Teichmann, A.T., et al. (1995) The influence of the dose of ethinylestradiol in oral contraceptives on follicle growth. Gynecological endocrinology: The Official Journal of the International Society of Gynecological Endocrinology, 9, 299-305. doi:10.3109/09513599509160463

[4] Qublan, H.S., et al. (2006) Ovarian cyst formation following GnRH agonist administration in IVF cycles: Incidence and impact. Human reproduction, 21, 640-644. doi:10.1093/humrep/dei371

[5] Nelson-Al, G.J. (2010) Congenital anomalies and benign conditions of the ovaries and fallopian tubes. In: Hacker, N.F., Gambone, J.C. and Hobel, C.J., Eds., Hacker and Moore's Essentials of Obstetrics and Gynecology, 5th Edition, Saunders, Philadelphia, 248-255.

[6] Beckmann, L.F. and Smith, R.P., et al. (2006) Ovarian and adnexal disease. In: Beckmann C.R.B., Ling F.W., Smith R.P., et al, Eds., Obstetrics and Gynecology, 5th Edition, Lippincott Williams \& Wilkins, Philadelphia, 464-476.

[7] Rossing, M.A., et al. (1994) Ovarian tumors in a cohort of infertile women. The New England Journal of Medicine, 331, 771-776. doi:10.1056/NEJM199409223311204

[8] Koonings, P.P., Campbell, K., Mishell, D.R. Jr. and Grimes, D.A. (1989) Relative frequency of primary ovarian neoplasms: A 10-year review. Obstet Gynecol, 74, 921926.

[9] Ayhan, A.B.O., Genc, C., Karamursel, B.S. and Ayhan, A. (2000) Mature cystic teratomas of the ovary: Case series from one institution over 34 years. European Journal of Obstetrics \& Gynecology and Reproductive, 88, 153157. doi:10.1016/S0301-2115(99)00141-4

[10] Caspi, B., et al. (1997) The growth pattern of ovarian dermoid cysts: A prospective study in premenopausal and postmenopausal women. Fertility and Sterility, 68, 501- 


\section{5. doi:10.1016/S0015-0282(97)00228-8}

[11] Hoo, W.L., et al. (2010) Expectant management of ultrasonically diagnosed ovarian dermoid cysts: Is it possible to predict outcome? Ultrasound in obstetrics \& gynecology: The Official Journal of the International Society of Ultrasound in Obstetrics and Gynecology, 36, 235-240. doi:10.1002/uog.7610

[12] O'Neill, K.E. and Cooper, A.R. (2011) The approach to ovarian dermoids in adolescents and young women. Journal of Pediatric and Adolescent Gynecology, 24, 176180. doi:10.1016/j.jpag.2010.11.006

[13] Gelbaya, T.A., et al. (2010) Management of endometrioma prior to IVF: Compliance with ESHRE guidelines. Reproductive Biomedicine Online, 21, 325-330. doi:10.1016/j.rbmo.2010.04.023

[14] ESHRE (2013) Management of women with endomertiosis. In press.

[15] Surrey, E.S. (2013) Endometriosis and assisted reproducetive technologies: Maximizing outcomes. Seminars in Reproductive Medicine, 31, 154-163.

[16] Tsoumpou, I., et al. (2009) The effect of surgical treatment for endometrioma on in vitro fertilization outcomes: A systematic review and meta-analysis. Fertility and Sterility, 92, 75-87. doi:10.1016/i.fertnstert.2008.05.049

[17] D.O.H.L.D. (2009) Key messages for ovarian cancer for health professionals.

[18] Ueland, F.R., et al. (2005) The accuracy of examination under anesthesia and transvaginal sonography in evaluating ovarian size. Gynecologic Oncology, 99, 400-403. doi:10.1016/j.ygyno.2005.06.030

[19] Ballard, K.D., et al. (2008) Can symptomatology help in the diagnosis of endometriosis? Findings from a national case-control study-Part 1. International Journal of $\mathrm{Ob}$ stetrics and Gynaecology, 115, 1382-1391. doi:10.1111/j.1471-0528.2008.01878.x

[20] Padilla, L.A., Radosevich, D.M. and Milad, M.P. (2000) Accuracy of the pelvic examination in detecting adnexal masses. Obstetrics and Gynecology, 96, 593-598. doi:10.1016/S0029-7844(00)00970-4

[21] Kahraman, K., et al. (2007) Extremely elevated serum CA-125 level as a result of unruptured unilateral endometrioma: The highest value reported. Fertility and sterility, 88, e15-e17. doi:10.1016/j.fertnstert.2006.12.076

[22] Zurawski, V.R., Jr., et al. (1988) Elevated serum CA 125 levels prior to diagnosis of ovarian neoplasia: Relevance for early detection of ovarian cancer. International Journal of Cancer, 42, 677-680. doi:10.1002/ijc.2910420507

[23] Van Calster, B., et al. (2007) Discrimination between benign and malignant adnexal masses by specialist ultrasound examination versus serum CA-125. Journal of the National Cancer Institute, 99, 1706-1714. doi:10.1093/jnci/djm199

[24] Im, S.S., et al. (2005) Validation of referral guidelines for women with pelvic masses. Obstetrics and Gynecology, 105, 35-41. doi:10.1097/01.AOG.0000149159.69560.ef

[25] Timmerman, D., et al. (2007) Inclusion of CA-125 does not improve mathematical models developed to distinguish between benign and malignant adnexal tumors.
Journal of Clinical Oncology: Official Journal of the American Society of Clinical Oncology, 25, 4194-200.

[26] Timmerman, D., et al. (2008) Simple ultrasound-based rules for the diagnosis of ovarian cancer. Ultrasound in obstetrics \& Gynecology: The Official Journal of the International Society of Ultrasound in Obstetrics and Gynecology, 31, 681-690. doi:10.1002/uog.5365

[27] Broekmans, F.J., et al. (2006) A systematic review of tests predicting ovarian reserve and IVF outcome. Human Reproduction Update, 12, 685-718. doi:10.1093/humupd/dml034

[28] De Vet, A., et al. (2002) Antimullerian hormone serum levels: A putative marker for ovarian aging. Fertility and Sterility, 77, 357-362. doi:10.1016/S0015-0282(01)02993-4

[29] Gnoth, C., et al. (2008) Relevance of anti-Mullerian hormone measurement in a routine IVF program. Human Reproduction, 23, 1359-1365. doi:10.1093/humrep/den 108

[30] Van Rooij, I.A., et al. (2005) Serum antimullerian hormone levels best reflect the reproductive decline with age in normal women with proven fertility: A longitudinal study. Fertility and Sterility, 83, 979-987. doi:10.1016/j.fertnstert.2004.11.029

[31] Almog, B., et al. (2011) Age-related normograms of serum antimullerian hormone levels in a population of infertile women: A multicenter study. Fertility and Sterility, 95, 2359-2363. doi:10.1016/i.fertnstert.2011.02.057

[32] Timmerman, D., et al. (1999) Subjective assessment of adnexal masses with the use of ultrasonography: An analysis of interobserver variability and experience. Ultrasound in Obstetrics \& Gynecology: The Official Journal of the International Society of Ultrasound in Obstetrics and Gynecology, 13, 11-16. doi:10.1046/j.1469-0705.1999.13010011.x

[33] Patel, M.D., et al. (1998) Cystic teratomas of the ovary: Diagnostic value of sonography. American Journal of Roentgenology, 171, 1061-1065. doi:10.2214/ajr.171.4.9762997

[34] Van Holsbeke, C., et al. (2010) Endometriomas: Their ultrasound characteristics. Ultrasound in Obstetrics \& Gynecology: The Official Journal of the International Society of Ultrasound in Obstetrics and Gynecology, 35, 730-740.

[35] Sassone, A.M., et al. (1991) Transvaginal sonographic characterization of ovarian disease: Evaluation of a new scoring system to predict ovarian malignancy. Obstetrics and Gynecology, 78, 70-76.

[36] Vuento, M.H., et al. (1995) Evaluation of ovarian findings in asymptomatic postmenopausal women with color doppler ultrasound. Cancer, 76, 1214-1218. doi:10.1002/1097-0142(19951001)76:7<1214::AID-CNC $\underline{\mathrm{R} 2820760718>3.0 . \mathrm{CO} ; 2-5}$

[37] Stein, S.M., et al. (1995) Differentiation of benign and malignant adnexal masses: Relative value of gray-scale, color doppler, and spectral doppler sonography. American Journal of Roentgenology, 164, 381-386. doi:10.2214/ajr.164.2.7839975 
[38] Dai, S.Y., et al. (2008) Does three-dimensional power doppler ultrasound improve the diagnostic accuracy for the prediction of adnexal malignancy? The Journal of Obstetrics and Gynaecology Research, 34, 364-370. doi:10.1111/j.1447-0756.2007.00702.x

[39] Guerriero, S., et al. (2007) Three-dimensional quantification of tumor vascularity as a tertiary test after B-mode and power doppler evaluation for detection of ovarian cancer. Journal of Ultrasound in Medicine, 26, 12711278.

[40] MacKenna, A., et al. (2000) Clinical management of functional ovarian cysts: A prospective and randomized study. Human Reproduction, 15, 2567-2569. doi:10.1093/humrep/15.12.2567

[41] Levine, D., et al. (2010) Management of asymptomatic ovarian and other adnexal cysts imaged at US: Society of radiologists in ultrasound consensus conference statement. Ultrasound Quarterly, 26, 121-131. doi:10.1097/RUQ.0b013e3181f09099

[42] Katz, L., et al. (2010) Pregnancy outcome of patients with dermoid and other benign ovarian cysts. Archives of Gynecology and Obstetrics, 281, 811-815. doi:10.1007/s00404-009-1158-1

[43] Grimes, D.A., et al. (2009) Oral contraceptives for functional ovarian cysts. The Cochrane Database of Systematic Reviews, 2, Article ID: CD006134.

[44] Karen P. and James E.W. (2002) In benign disorders of the ovaries \& oviducts. Current Obstetrics \& Gynecologic diagnosis \& treatment, 9th Edition, McGrak Hill, pp. 708-715.

[45] Holt, V.L., Cushing-Haugen, K.L. and Daling, J.R. (2003) Oral contraceptives, tubal sterilization, and functional ovarian cyst risk. Obstetrics and Gynecology, 102, 252258. doi:10.1016/S0029-7844(03)00572-6

[46] Kim, S.H., et al. (2013) Update on the treatment of endometriosis. Clinical and Experimental Reproductive Medicine, 40, 55-59. doi:10.5653/cerm.2013.40.2.55

[47] Raffi, F., Shaw, R.W. and Amer, S.A. (2012) National survey of the current management of endometriomas in women undergoing assisted reproductive treatment. $\mathrm{Hu}$ man Reproduction, 27, 2712-2719. doi:10.1093/humrep/des195

[48] Tavmergen, E., Ulukus, M. and Goker, E.N. (2007) Long-term use of gonadotropin-releasing hormone analogues before IVF in women with endometriosis. Current Opinion in Obstetrics \& Gynecology, 19, 284-288. doi:10.1097/GCO.0b013e3281053a52

[49] Surrey, E.S., et al. (2002) Effect of prolonged gonadotropin-releasing hormone agonist therapy on the outcome of in vitro fertilization-embryo transfer in patients with endometriosis. Fertility and Sterility, 78, 699-704. doi:10.1016/S0015-0282(02)03373-3
[50] Ben-Ami, M., et al. (1993) Management of functional ovarian cysts after induction of ovulation. A randomized prospective study. Acta Obstetricia et Gynecologica Scandinavica, 72, 396-397. doi:10.3109/00016349309021121

[51] Steinkampf, M.P., Hammond, K.R. and Blackwell, R.E. (1990) Hormonal treatment of functional ovarian cysts: A randomized, prospective study. Fertility and Sterility, 54, 775-777.

[52] Turan, C., et al. (1994) Expectant management of functional ovarian cysts: An alternative to hormonal therapy. International Journal of Gynaecology and Obstetrics, 47, 257-260. doi:10.1016/0020-7292(94)90570-3

[53] Alcazar, J.L., et al. (2005) Is expectant management of sonographically benign adnexal cysts an option in selected asymptomatic premenopausal women? Human Reproduction, 20, 3231-3234.

[54] Mais, V., et al. (2003) No recurrence of mature ovarian teratomas after laparoscopic cystectomy. BJOG: An International Journal of Obstetrics and Gynaecology, 110, 624-626. doi:10.1046/j.1471-0528.2003.02040.x

[55] Yuen, P.M., et al. (1997) A randomized prospective study of laparoscopy and laparotomy in the management of benign ovarian masses. American Journal of Obstetrics and Gynecology, 177, 109-114. doi:10.1016/S0002-9378(97)70447-2

[56] Panici, P.B., et al. (2007) Minilaparotomy versus laparoscopy in the treatment of benign adnexal cysts: A randomized clinical study. European Journal of Obstetrics, Gynecology, and Reproductive Biology, 133, 218-222. doi:10.1016/j.ejogrb.2006.05.019

[57] Fanfani, F., et al. (2004) A prospective randomized study of laparoscopy and minilaparotomy in the management of benign adnexal masses. Human Reproduction, 19, 23672371. doi:10.1093/humrep/deh413

[58] Medeiros, L.R., et al. (2008) Laparoscopy versus laparotomy for benign ovarian tumor: A systematic review and meta-analysis. International Journal of Gynecological Cancer, 18, 387-399.

[59] Garcea, N., et al. (1996) Surgical treatment of ovarian cysts: Economic analysis as a means for the evaluation of alternative technics. Results of a biennial study. Minerva Ginecologica, 48, 77-83.

[60] Zanetta, G., et al. (1996) Role of puncture and aspiration in expectant management of simple ovarian cysts: A randomised study. British Medical Journal, 313, 1110-1113. doi:10.1136/bmj.313.7065.1110

[61] Marana, R., et al. (1996) Operative laparoscopy for ovarian cysts. Excision vs. aspiration. The Journal of Reproductive Medicine, 41, 435-438. 